\title{
https://tecnohumanismo.online
}

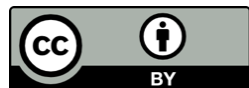

\section{La administracion del tiempo y la satisfaccion laboral de los servidores administrativos de la municipalidad distrital de Grocio Prado, año 2016}

Proposal for Emotional Competencies and conflict resolution in university students Proposta de Competências Emocionais e Resolução de Conflitos em Estudantes Universitários.

\section{ARTÍCULO GENERAL}

Ericka Janet Villamares Hernández

Universidad Nacional San Luis
Gonzaga de Ica, Ica - Perú

\section{Jorge Luis De La Cruz Saravia}

Universidad Nacional San Luis Gonzaga de Ica, Ica - Perú
Jorge Luis De La Cruz Martinez

Universidad Nacional San Luis

Gonzaga de Ica, Ica - Perú

Recibido 16 de Diciembre 2020 | Arbitrado y aceptado 06 de Enero 2021 | Publicado el 01 de Marzo 2021

\section{RESUMEN}

La satisfacción laboral influye positivamente en la productividad, el cumplimiento de metas y el tiempo de permanencia en una organización. Un empleado satisfecho es rentable. Y la relación entre la administración del tiempo y la satisfacción laboral es un tema ampliamente estudiado precisamente por su importancia para las organizaciones

Objetivo: Verificar que la Administración del Tiempo tiene una relación significativa con la Satisfacción Laboral de los servidores administrativos de la Universidad Nacional San Luis Gonzaga de Ica, año 2016.

Material y métodos: La investigación corresponde a un nivel correlacional. -Es analítica y aplicativa. Sobre una muestra de 269 trabajadores administrativos de la Universidad Nacional San Luis Gonzaga de Ica.

Resultados: La Administración del tiempo tiene una relación significativa con la Satisfacción laboral de los servidores administrativos de la Universidad Nacional San Luis Gonzaga de Ica con una muy buena correlación de $82.6 \%$. La planeación del tiempo se relaciona significativamente con la Satisfacción Laboral de los servidores administrativos de la Universidad Nacional San Luis Gonzaga de Ica con una buena correlación de $75.9 \%$. La priorización del tiempo se relaciona directamente con la Satisfacción Laboral de los servidores administrativos de la Universidad Nacional San Luis Gonzaga de Ica con una buena correlación de $79.4 \%$. La organización del tiempo se relaciona directamente con la Satisfacción Laboral de los servidores administrativos de la Universidad Nacional San Luis Gonzaga de Ica con una buena correlación de $75.0 \%$. El control de actividades tiene una relación significativa con la Satisfacción Laboral de los servidores administrativos de la Universidad Nacional San Luis Gonzaga de Ica con una buena correlación de $64.2 \%$. Conclusiones. Existe una muy buena correlación entre la administración del trabajo con la satisfacción laboral. Un trabajo bien planificado genera un trabajador satisfecho.

PALABRAS CLAVES: Administración tiempo, satisfacción laboral.

\section{ABSTRACT}

Job satisfaction positively influences productivity, goal achievement and the time spent in an organization. A satisfied employee is profitable. And the relationship between time management and job satisfaction is a subject widely studied precisely because of its importance for organizations.

Objective: Verify that Time Management has a significant relationship with the Job Satisfaction of the administrative servers of the San Luis Gonzaga de Ica National University, year 2016.

Material and methods: The research corresponds to a correlational level. -It is analytical and applicative. On a sample of 269 administrative workers from the San Luis Gonzaga National University of Ica.

Results: Time management has a significant relationship with the job satisfaction of the administrative servers of the San Luis Gonzaga National University of Ica with a very good correlation of $82.6 \%$. Time planning is significantly related to the Job Satisfaction of the administrative servers of the San Luis Gonzaga National University of Ica with a good correlation of $75.9 \%$. The prioritization of time is directly related to the Job Satisfaction of the administrative servers of the San Luis Gonzaga National University of Ica with a good correlation of $79.4 \%$. The organization of time is directly related to the Job Satisfaction of the administrative servers of the San Luis Gonzaga National University of Ica with a good correlation of $75.0 \%$. The control of activities has a significant relationship with the Job Satisfaction of the administrative servers of the San Luis Gonzaga National University of Ica with a good correlation of $64.2 \%$. conclusions. There is a very good correlation between job management and job satisfaction. A well-planned job generates a satisfied worker.

KEY WORDS: Time management, job satisfaction.

\section{RESUMO}

A satisfação no trabalho influencia positivamente a produtividade, o alcance de metas e o tempo gasto em uma organização. Um funcionário satisfeito é rentável. E a relação entre gestão do tempo e satisfação no trabalho é um assunto amplamente estudado justamente por sua importância para as organizações.

Objetivo: Verificar se a Gestão do Tempo tem uma relação significativa com a Satisfação no Trabalho dos servidores administrativos da Universidade Nacional San Luis Gonzaga de Ica, ano 2016.

Material e métodos: A pesquisa corresponde a um nível correlacional. -É analítico e aplicativo. Em uma amostra de 269 trabalhadores administrativos da Universidade Nacional San Luis Gonzaga de Ica.

Resultados: A gestão do tempo tem uma relação significativa com a satisfação no trabalho dos servidores administrativos da Universidade Nacional San Luis Gonzaga de Ica com uma correlação muito boa de $82,6 \%$. O planejamento do tempo está significativamente relacionado com a Satisfação no Trabalho dos servidores administrativos da Universidade Nacional San Luis Gonzaga de Ica com uma boa correlação de $75,9 \%$. A priorização do tempo está diretamente relacionada com a Satisfação no Trabalho dos servidores administrativos da Universidade Nacional San Luis Gonzaga de Ica com uma boa correlação de $79,4 \%$. A organização do tempo está diretamente relacionada com a Satisfação no Trabalho dos servidores administrativos da Universidade Nacional San Luis Gonzaga de Ica com uma boa correlação de $75,0 \%$. O controle das atividades tem relação significativa com a Satisfação no Trabalho dos servidores administrativos da Universidade Nacional San Luis Gonzaga de Ica com uma boa correlação de $64,2 \%$. conclusões. Existe uma correlação muito boa entre a gestão do trabalho e a satisfação no trabalho. Um trabalho bem planejado gera um trabalhador satisfeito.

PALAVRAS-CHAVE: Gestão do tempo, satisfação no trabalho. 


\section{Introducción}

El presente proyecto de investigación sobre "La Administración del Tiempo y la Satisfacción Laboral de los Servidores Administrativos de la Universidad San Luis Gonzaga de Ica, Año 2016”.

El problema objeto de la presente investigación queda planteado por la siguiente interrogante: ¿De qué manera la Administración del Tiempo se relaciona con la Satisfacción Laboral de los Servidores Administrativos de la Universidad San Luis Gonzaga de Ica, Año 2016? al cual se plantea una hipótesis basada en una relación directa entre las variables del problema indicado. La variable independiente del problema es la Administración del Tiempo y la Variable dependiente es la Satisfacción Laboral.

Las técnicas a utilizarse en la recolección de información que nos permita contrastar la hipótesis son el análisis documental, la encuesta estructurada, y la observación estructurada, para el caso del tratamiento de la información utilizaremos los métodos: inductivo-deductivo, de análisis y síntesis, la inferencia estadística y otras técnicas estadísticas.

Este proyecto va a tratar de contribuir en la búsqueda de alternativas de solución a la problemática propuesta que permita el análisis de la Administración del Tiempo de la labor de los Servidores Administrativos de dicha municipalidad, como elemento fundamental de la Satisfacción Laboral de los mismos, presentando alternativas en forma integral y global para el mejor cumplimiento de la Misión, Visión, Objetivos personales en la satisfacción de su calidad de vida.

La satisfacción laboral influye positivamente en la productividad, el cumplimiento de metas y el tiempo de permanencia en una organización. Un empleado satisfecho es rentable.

Acciones como la de mantener contento y en excelentes condiciones de trabajo, son las que contribuyen a fortalecer la mística, el sentimiento de pertenencia y fidelidad del colaborador con la empresa. Para Fabián Bermeo, coordinador nacional de Talento Humano de la Universidad Politécnica Salesiana, afirmó que "esa mística no depende solo de la predisposición del empleado, sino también de lo que hace la empresa para motivarlo". 
Cuando un empleado se siente satisfecho con el tiempo dedicado a su trabajo, hace lo que le gusta y está orgulloso de trabajar en la compañía, por lo que las posibilidades de que la abandonen son menores.

Para esta organización la mística se identifica con indicadores claros. La confianza (con los líderes, los compañeros, los procesos y con la empresa), la camaradería (para facilitar el entendimiento) y el orgullo (por la empresa y las funciones que desempeña).

Para los expertos, un empleado con mística es un buen negocio, porque cumplen los objetivos, tiene planes de quedarse y, además, hace una suerte de apostolado entre sus colegas.

Se dice, hay alta rotación de personal por falta de esa mística, en algunos casos, como el sector educación, llega al $40 \%$ anual o en las ventas al por menor al $30 \%$, cuando lo deseable es del 7 u $8 \%$.

Pero hay criterios distintos. El experto en talento humano, Cristian Castillo, cree que la mística se da cuando se conjugan particularidades como una buena propuesta económica, predisposición, opciones de desarrollo y conocimiento.

Se cree que la mística es consecuencia de alguna acción de la compañía para lograrla. La unión de estas condiciones no es común, sino excepcional.

En los niveles de satisfacción no se pueden dar un criterio veras ya que no se ha realizado anteriormente investigaciones de este tipo para conocer índices acertados que puedan tener satisfechos a los colaboradores de la empresa.

Esta investigación pretende ahondar en las interacciones que tienen lugar en algunos de los procesos humanos de la organización, y su consecuente efecto en la productividad, pues si bien ésta se encuentra multideterminada por diversos factores o elementos (tecnológicos, financieros, mercadotécnicos, etc.), es la influencia de los procesos humanos los que parecen ejercer el mayor efecto para lograrla. Naturalmente, semejante propósito tiene que limitarse a una "totalidad" menor de la totalidad que implica la organización, al menos como estrategia de abordaje, debido a la complejidad del fenómeno. 


\section{MATERIALES Y MÉTODOS}

\section{Diseño y población de estudio}

Se consideró un estudio descriptivo correlacional, observacional, de carácter cuantitativo por su naturaleza numérica. El nivel fue descriptivo y el diseño correlacional. La población estudiada fueron los servidores administrativos registrados en la planilla oficial de la Universidad, quienes proporcionaron la información pertinente al estudio.

La muestra estuvo determinada por 269 servidores administrativos (según datos proporcionados por la Oficina de Personal de la Universidad Nacional San Luis Gonzaga de Ica). Los datos obtenidos fueron ingresados a la base de datos SPSS 22.

\section{Variables y mediciones}

Se incluyeron un total de 269 trabajadores en el estudio, a los cuales se les aplicó un cuestionario para medir la Administración del tiempo y se aplicó la técnica de observación para evaluar la Satisfacción laboral. Para efectos de esta investigación el cuestionario se sometió a juicio de expertos para determinar la validez del contenido, de constructo y de criterio, y luego se realizó una prueba piloto para probar el grado de comprensión por los participantes. Para identificar las características de la Administración del tiempo en los servidores administrativos se empleó un cuestionario con 25 interrogantes, utilizando la escala de Likert, siendo el puntaje más bajo ( 1 = Rara vez $)$, el puntaje medio $(2=$ A veces $)$ y el puntaje más alto $(3=$ Frecuentemente $)$

\section{Análisis estadístico}

Se construyeron tablas y gráficos estadísticos y calcularán estadígrafos y medidas de tendencia central para facilitar el análisis e interpretación de los resultados. Para el análisis e interpretación de la información, se utilizó básicamente el estadístico descriptivo, y, para la prueba de hipótesis se utilizó la inferencia estadística.

\section{Consideraciones éticas}

La investigación mantuvo en todo momento la confidencialidad de los participantes y de los datos proporcionados por la Oficina de Personal de la Universidad Nacional San Luis Gonzaga de Ica. 


\section{RESULTADOS}

\section{Tabla 1}

Satisfacción laboral de los servidores administrativos

\begin{tabular}{lcc}
\hline Satisfacción & Nivel de satisfacción & Porcentaje \\
\hline Índice de satisfacción & 87.5 & $87.5 \%$ \\
Índice de insatisfacción & 12.5 & $12.5 \%$ \\
Calificación máxima & 100 & $100 \%$ \\
\hline
\end{tabular}

Fuente: Universidad San Luis Gonzaga de Ica

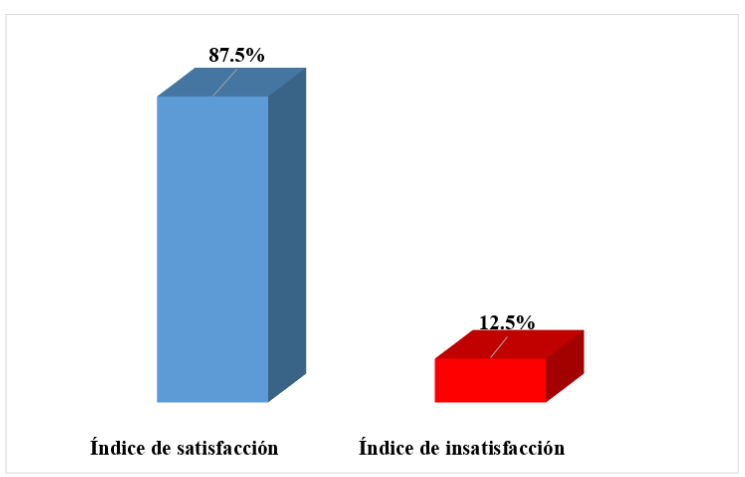

Figura 1. Satisfacción laboral de los servidores administrativos.

Los resultados arrojan un $87.5 \%$ de satisfacción laboral en los servidores administrativos de la Universidad San Luis Gonzaga de Ica.

Tabla 2

Administración del tiempo y su relación con la satisfacción laboral

\begin{tabular}{lccc}
\hline $\begin{array}{c}\text { ADMINISTRACIÓN } \\
\text { DEL TIEMPO }\end{array}$ & $\begin{array}{c}\text { Media de } \\
\text { satisfacción }\end{array}$ & No & IC 95\% \\
\hline RARA VEZ & 63.50 & $02(0.7 \%)$ & $60.6-66.4$ \\
A VECES & 77.06 & $121(45.0 \%)$ & $75.7-78.4$ \\
FRECUENTEMENTE & 96.95 & $146(54.3 \%)$ & $95.5-98.4$ \\
Total & 87.75 & 269 & \\
\hline
\end{tabular}

Fuente: Universidad San Luis Gonzaga de Ica 


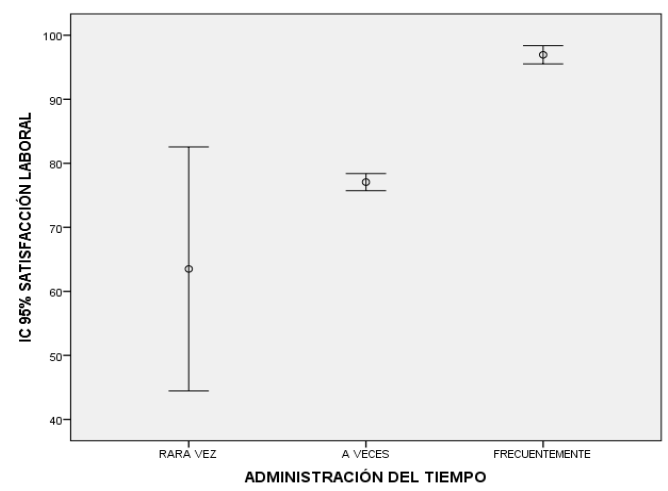

Figura 4. Priorización de actividades y su relación con la satisfacción laboral.

Los resultados muestran que los que rara vez priorizan sus actividades tienen un índice de satisfacción laboral promedio de 62.56, en los que lo hacen a veces, el promedio de satisfacción laboral es de 80.22 y los que los hacen frecuentemente el promedio de satisfacción laboral es de 97.70, en los servidores administrativos de la Universidad San Luis Gonzaga de Ica.

\section{Tabla 5}

\section{Organización del tiempo y su relación con la satisfacción laboral}

\begin{tabular}{lccc}
\hline $\begin{array}{c}\text { ORGANIZACIÓN DEL } \\
\text { TIEMPO }\end{array}$ & $\begin{array}{c}\text { Media de } \\
\text { satisfacción }\end{array}$ & No & IC 95\% \\
\hline RARA VEZ & 62.14 & $07(2.6 \%)$ & $60.7-63.6$ \\
A VECES & 74.84 & $74(27.5 \%)$ & $73.7-76.0$ \\
FRECUENTEMENTE & 93.79 & $188(69.9 \%)$ & $92.3-95.2$ \\
Total & 87.75 & 269 & \\
\hline
\end{tabular}

Fuente: Universidad San Luis Gonzaga de Ica

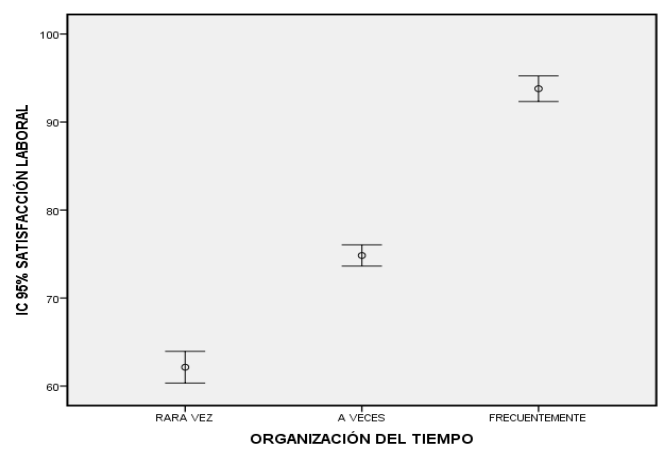

Figura 5. Organización del tiempo y su relación con la satisfacción laboral 
Los resultados muestran que los que rara vez organizan su tiempo tienen un índice de satisfacción laboral promedio de 62.14 , en los que lo hacen a veces, el promedio de satisfacción laboral es de 74.84 y los que los hacen frecuentemente el promedio de satisfacción laboral es de 93.79, en los servidores administrativos de la Universidad San Luis Gonzaga de Ica.

Tabla 6

Control de actividades y su relación con la satisfacción laboral

\begin{tabular}{lccc}
\hline \multicolumn{1}{c}{$\begin{array}{c}\text { CONTROL DE } \\
\text { ACTIVIDADES }\end{array}$} & $\begin{array}{c}\text { Media de } \\
\text { satisfacción }\end{array}$ & No & IC 95\% \\
\hline RARA VEZ & 72.67 & $09(3.3 \%)$ & $67.8-77.5$ \\
A VECES & 76.40 & $78(29.0 \%)$ & $74.1-78.7$ \\
FRECUENTEMENTE & 93.37 & $182(67.7 \%)$ & $91.9-94.8$ \\
Total & 87.75 & 269 & \\
\hline
\end{tabular}

Fuente: Universidad San Luis Gonzaga de Ica

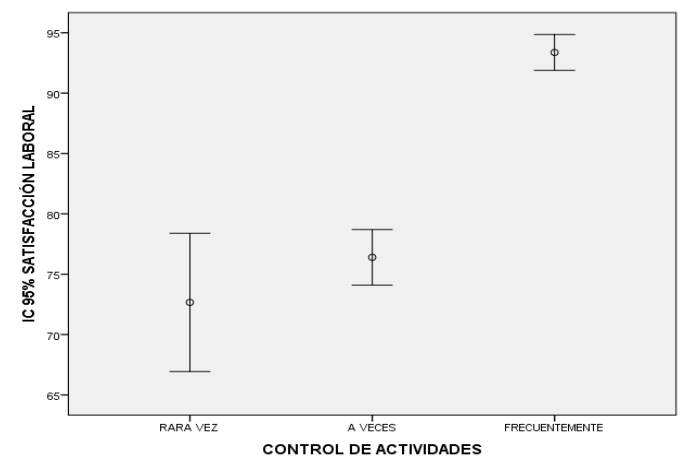

Figura 6. Control de actividades y su relación con la satisfacción laboral

Los resultados muestran que los que rara vez controlan sus actividades tienen un índice de satisfacción laboral promedio de 72.67, en los que lo hacen a veces, el promedio de satisfacción laboral es de 76.40 y los que los hacen frecuentemente el promedio de satisfacción laboral es de 93.37, en los servidores administrativos de la Universidad San Luis Gonzaga de Ica. 


\section{Contrastación de Hipótesis}

\section{Prueba de Hipótesis General}

\section{1.- Formulación de la hipótesis}

Ha: La Administración del Tiempo tiene relación significativa con la Satisfacción laboral de los servidores administrativos de la Universidad San Luis Gonzaga de Ica, Año 2016.

Ho: La Administración del Tiempo no tiene relación significativa con la Satisfacción laboral de los servidores administrativos de la Universidad San Luis Gonzaga de Ica, Año 2016.

2.- Nivel de confianza: $95 \%$ : 0.05 de confianza

\section{3.- Estadístico de prueba}

\begin{tabular}{|c|c|c|}
\hline & & $\begin{array}{c}\text { ADMINISTRACIÓN } \\
\text { DEL TIEMPO } \\
\end{array}$ \\
\hline Rho de & SATISFACCIÓN Coeficiente de correlación & 0.826 \\
\hline Spearman & $\begin{array}{ll}\text { LABORAL } & \text { Sig. (bilateral) } \\
\mathrm{N}\end{array}$ & $\begin{array}{r}0.000 \\
269\end{array}$ \\
\hline
\end{tabular}

4.- Determinación del $p$ valor: $p=0.000$

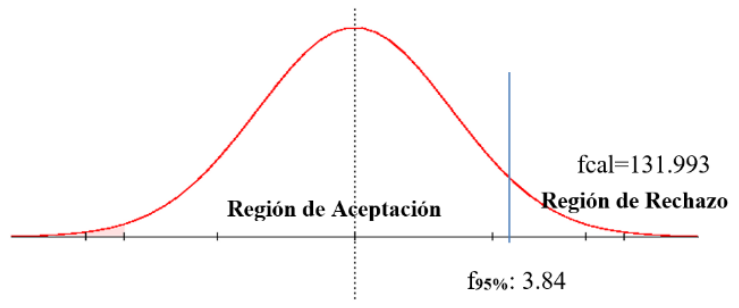

\section{5.- Decisión}

Se rechaza la hipótesis nula y se acepta la hipótesis alterna, es decir: La Administración del tiempo tiene relación significativa con la Satisfacción laboral de los servidores administrativos de la Universidad San Luis Gonzaga de Ica, Año 2016 con una muy buena correlación de $82.6 \%$. 


\section{Prueba de Hipótesis Específica 1}

\section{1.- Formulación de la hipótesis}

Ha: La planeación del tiempo se relaciona significativamente con la Satisfacción Laboral de los servidores administrativos de la Universidad San Luis Gonzaga de Ica, Año 2016.

Ho: La planeación del tiempo no se relaciona significativamente con la Satisfacción Laboral de los servidores administrativos de la Universidad San Luis Gonzaga de Ica, Año 2016.

2.- Nivel de confianza: 95\%: 0.05 de confianza

\section{3.- Estadístico de prueba}

\begin{tabular}{lllr}
\hline & & PLANEACIÓN \\
\hline Rho de & SATISFACCIÓN & Coeficiente de correlación & 0.759 \\
Spearman & LABORAL & Sig. (bilateral) & 0.000 \\
& & N & 269 \\
\hline
\end{tabular}

\section{4.- Determinación del $p$ valor $=\mathbf{0 . 0 0 0}$}

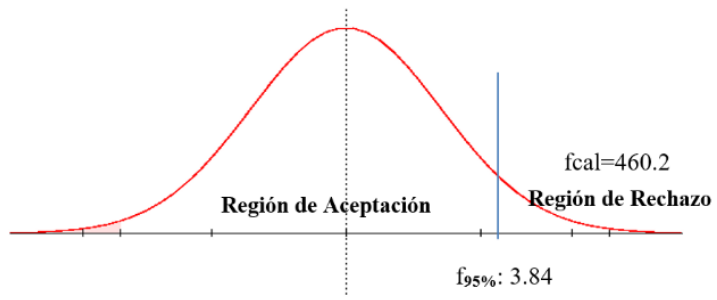

\section{5.- Decisión}

Se rechaza la hipótesis nula y se acepta la hipótesis alterna, es decir: La planeación del tiempo se relaciona significativamente con la Satisfacción Laboral de los servidores administrativos de la Universidad San Luis Gonzaga de Ica, Año 2016, con una buena correlación de $75.9 \%$.

\section{Prueba de Hipótesis Específica 2}

\section{1.- Formulación de la hipótesis}

Ha: La priorización del tiempo se relaciona directamente con la Satisfacción Laboral de los servidores administrativos de la Universidad San Luis Gonzaga de Ica, Año 2016 
Ho: La priorización del tiempo no se relaciona directamente con la Satisfacción Laboral de los servidores administrativos de la Universidad San Luis Gonzaga de Ica, Año 2016

2.- Nivel de confianza: 95\%: 0.05 de confianza

\section{3.- Estadístico de prueba}

\begin{tabular}{lllr}
\hline & & PRIORIZACIÓN \\
\hline Rho de & SATISFACCIÓN & Coeficiente de correlación & 0.794 \\
Spearman & LABORAL & Sig. (bilateral) & 0.000 \\
& & N & 269 \\
\hline
\end{tabular}

4.- Determinación del p valor: 0.000

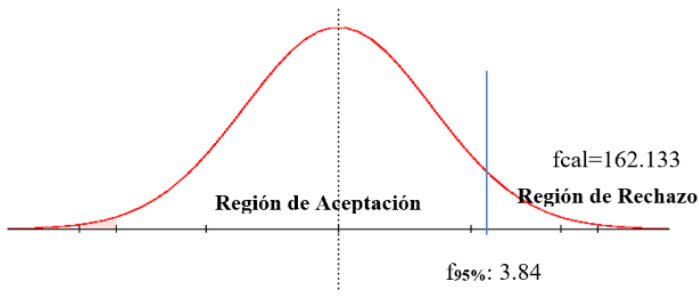

\section{5.- Decisión}

Se rechaza la hipótesis nula y se acepta la hipótesis alterna, es decir: La priorización del tiempo se relaciona directamente con la Satisfacción Laboral de los servidores administrativos de la Universidad San Luis Gonzaga de Ica, Año 2016, con una buena correlación de $79.4 \%$.

\section{Prueba de Hipótesis Específica 3}

\section{1.- Formulación de la hipótesis}

Ha: La organización del tiempo se relaciona directamente con la Satisfacción Laboral de los servidores administrativos de la Universidad San Luis Gonzaga de Ica, Año 2016

Ho: La organización del tiempo no se relaciona directamente con la Satisfacción Laboral de los servidores administrativos de la Universidad San Luis Gonzaga de Ica, Año 2016

2.- Nivel de confianza: 95\%: 0.05 de confianza 


\section{3.- Estadístico de prueba}

\begin{tabular}{lllr}
\hline & & ORGANIZACIÓN \\
\hline Rho de & SATISFACCIÓN & Coeficiente de correlación & 0.750 \\
Spearman & LABORAL & Sig. (bilateral) & 0.000 \\
& & $\mathrm{~N}$ & 269 \\
\hline
\end{tabular}

4.- Determinación del p valor: 0.000

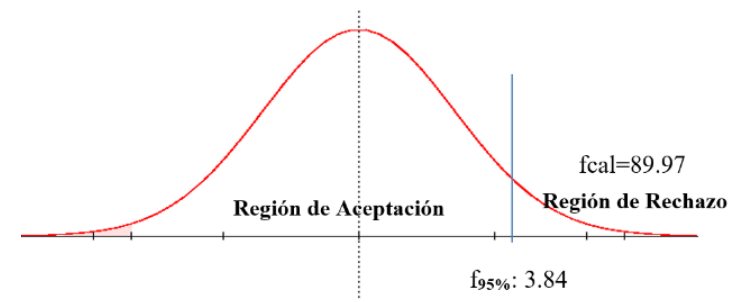

\section{5.- Decisión}

Se rechaza la hipótesis nula y se acepta la hipótesis alterna, es decir: La organización del tiempo se relaciona directamente con la Satisfacción Laboral de los servidores administrativos de la Universidad San Luis Gonzaga de Ica, Año 2016, con una buena correlación de $75.0 \%$.

\section{Prueba de Hipótesis Específica 4}

\section{1.- Formulación de la hipótesis}

Ha: El control de actividades tiene una relación significativa con la Satisfacción Laboral de los servidores administrativos de la Universidad San Luis Gonzaga de Ica, Año 2016

Ho: El control de actividades no tiene una relación significativa con la Satisfacción Laboral de los servidores administrativos de la Universidad San Luis Gonzaga de Ica, Año 2016

2.- Nivel de confianza: 95\%: 0.05 de confianza

\section{3.- Estadístico de prueba}

\begin{tabular}{lllr}
\hline & & & CONTROL DE \\
& & & ACTIVIDADES \\
\hline Rho de & SATISFACCIÓN & Coeficiente de correlación & 0.642 \\
Spearman & LABORAL & Sig. (bilateral) & 0.000 \\
& & $\mathrm{~N}$ & 269 \\
\hline
\end{tabular}




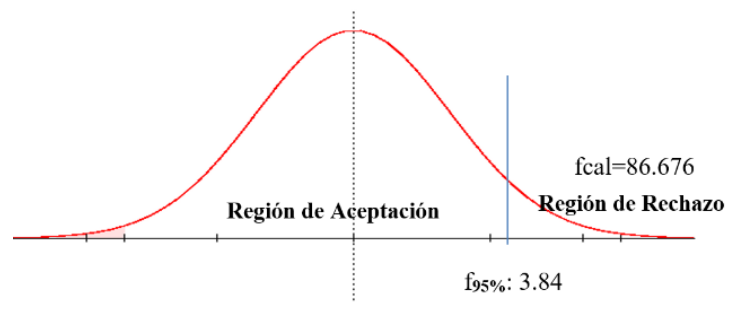

\section{5.- Decisión}

Se rechaza la hipótesis nula y se acepta la hipótesis alterna, es decir: El control de actividades tiene una relación significativa con la Satisfacción Laboral de los servidores administrativos de la Universidad San Luis Gonzaga de Ica, Año 2016, con una buena correlación de $64.2 \%$.

\section{Figura 2. Administración del tiempo y su relación con la satisfacción laboral}

Los resultados muestran que los que rara vez administran su tiempo, tienen un índice de satisfacción laboral promedio de 63.5, en los que lo hacen a veces el promedio de satisfacción laboral es de 77.06 y los que los hacen frecuentemente el promedio de satisfacción laboral es de 96.95, en los servidores administrativos de la Universidad San Luis Gonzaga de Ica.

\section{Tabla 3}

Planeación del tiempo y su relación con la satisfacción laboral

\begin{tabular}{lccc}
\hline $\begin{array}{c}\text { PLANEACIÓN DEL } \\
\text { TIEMPO }\end{array}$ & $\begin{array}{c}\text { Media de } \\
\text { satisfacción }\end{array}$ & N & IC 95\% \\
\hline RARA VEZ & 69.60 & $53(19.7 \%)$ & $61.3-77.9$ \\
A VECES & 89.43 & $175(65.0 \%)$ & $74.1-104.8$ \\
FRECUENTEMENTE & 104.07 & $41(15.3 \%)$ & $83.3-124.9$ \\
Total & 87.75 & 269 & \\
\hline
\end{tabular}

Fuente: Universidad San Luis Gonzaga de Ica

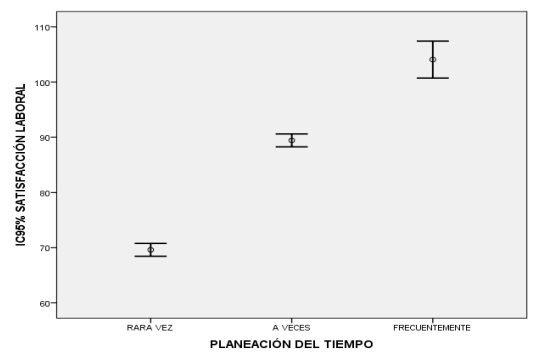

Figura 3. Planeación del tiempo y su relación con la satisfacción laboral. 
Los resultados muestran que los que rara vez planean su tiempo tienen un índice de satisfacción laboral promedio de 69.6, en los que lo hacen a veces, el promedio de satisfacción laboral es de 89.43 y los que los hacen frecuentemente el promedio de satisfacción laboral es de 104.07, en los servidores administrativos de la Universidad San Luis Gonzaga de Ica.

Tabla 4

Priorización de actividades y su relación con la satisfacción laboral

\begin{tabular}{lccc}
\hline $\begin{array}{c}\text { PRIORIZACIÓN DE } \\
\text { ACTIVIDADES }\end{array}$ & $\begin{array}{c}\text { Media de } \\
\text { satisfacción }\end{array}$ & No & IC 95\% \\
\hline RARA VEZ & 62.56 & $09(3.3 \%)$ & $61.3-63.8$ \\
A VECES & 80.22 & $135(50.2 \%)$ & $78.8-81.7$ \\
FRECUENTEMENTE & 97.70 & $125(46.5 \%)$ & $99.2-99.2$ \\
Total & 87.75 & 269 & \\
\hline
\end{tabular}

Fuente: Universidad San Luis Gonzaga de Ica

\section{DISCUSION}

El estudio sobre la relación que existe entre la administración del tiempo con la satisfacción laboral, mostró los siguientes resultados.

En la tabla $\mathrm{N}^{\circ} 01$; se observa que el nivel de satisfacción laboral general percibida en los servidores administrativos de la Universidad San Luis Gonzaga de Ica, año 2016, es de $87.5 \%$ lo que indica que es un nivel aceptable lo que se debería al ambiente laboral que se vive en la universidad y a las facilidades laborales que se presentan. Al respecto en Lima Marquina Vergara, Carlos Francesco (2011) encuentra solo 51.3\% de satisfacción laboral en la Universidad Privada de Lima, siendo las políticas de la Universidad las que se relacionan con la insatisfacción en un $73 \%$.

En la tabla $\mathrm{N}^{\circ} 02$; se relaciona la administración del tiempo con la satisfacción laboral, encontrándose una muy buena correlación del orden del $82.6 \%$ lo que indica que cuanto mejor se administra el tiempo repercute favorablemente en la satisfacción laboral, observándose que menos del 1\% lo hace rara vez, el $45 \%$ lo hace a veces y el 54\% lo hace frecuentemente, por lo que debe recomendarse una buena administración del tiempo en el grupo de trabajadores que lo hace rara vez o a veces. Al respecto Rasouli A. Hossenian S. Dokanee F. (2012) encontró en Irán una relación significativa la dureza y la satisfacción laboral. 
En la tabla $\mathrm{N}^{\circ} 03$; se relaciona la dimensión de planeación de la administración del tiempo con la satisfacción laboral, encontrándose que dicha relación tiene una buena correlación del orden del $75.9 \%$ mostrándose que a mejor planeación del tiempo se relaciona con una mayor satisfacción laboral, existiendo un $65 \%$ que lo realiza a veces y $19.7 \%$ que lo hace rara vez, por lo que debería recomendarse que a planeación del tiempo debe hacerse rutinariamente por los efectos favorables sobre la satisfacción laboral. Wong Prada, Claudia Patricia, Yataco Valdez, Palmer Edwin (2011) en Chincha encuentra una buena correlación entre la administración del tiempo con el rendimiento académico en los alumnos.

En la Tabla $N^{\circ} 04$ se relaciona la dimensión de priorización de la administración del trabajo con la satisfacción laboral hallándose que esta relación tiene una buena correlación del orden del 79.4\%, sin embargo, la priorización del tiempo en los servidores administrativos se produce frecuentemente en el $46.5 \%$ de trabajadores, a veces en el $50.2 \%$ y rara vez en el $3.3 \%$ por lo que es de necesidad recomendar realizar esta práctica frecuentemente por tener una buena repercusión en la satisfacción laboral.

En la tabla $\mathrm{N}^{\circ} 05$ se relaciona la dimensión organización del tiempo con la satisfacción laboral presentando una buena correlación pues ello se produce en el 75\% de casos, pero esta cualidad es desarrollada frecuentemente en el $69.9 \%$ der los trabajadores a veces el $27.5 \%$ y rara vez en el $2.6 \%$, a pesar de su alto grado de práctica de esta cualidad se debe recomendar a los demás trabajadores que lo practiquen pues ello repercutirá favorablemente en la satisfacción laboral. Galicia Corona, Vianka Leticia (2005) demostró que si los alumnos administran bien su tiempo pueden desarrollar satisfactoriamente sus tesis.

En la tabla $\mathrm{N}^{\circ} 06$ se relaciona la última dimensión de la administración del tiempo en el trabajo ello es el control de actividades y su relación con la satisfacción laboral encontrándose que se relaciona favorablemente en el orden del $64.2 \%$, sin embargo, es la dimensión que menos lo practican los trabajadores, pues el $67.7 \%$ lo realizan frecuentemente, $29 \%$ a veces y $3.3 \%$ rara vez. Es pues de necesidad que se debe recomendar el control de actividades pues ello generaría menos estrés y por lo tanto mayor satisfacción laboral. Al respecto Álvarez F. D. (2007) en Lima encuentra que las fuentes de presión laboral y la satisfacción laboral se relacionan de manera inversa. 


\section{CONCLUSIONES}

1. La Administración del tiempo tiene una relación significativa con la Satisfacción laboral de los servidores administrativos de la Universidad San Luis Gonzaga de Ica, Año 2016, con una muy buena correlación de 82.6\%.

2. La planeación del tiempo se relaciona significativamente con la Satisfacción Laboral de los servidores administrativos de la Universidad San Luis Gonzaga de Ica, Año 2016, con una buena correlación de 75.9\%.

3. La priorización del tiempo se relaciona directamente con la Satisfacción Laboral de los servidores administrativos de la Universidad San Luis Gonzaga de Ica, Año 2016, con una buena correlación de 79.4\%.

4. La organización del tiempo se relaciona directamente con la Satisfacción Laboral de los servidores administrativos de la Universidad San Luis Gonzaga de Ica, Año 2016, con una buena correlación de 75.0\%.

5. El control de actividades tiene una relación significativa con la Satisfacción Laboral de los servidores administrativos de la Universidad San Luis Gonzaga de Ica, Año 2016, con una buena correlación de 64.2\%.

\section{REFERENCIAS BIBLIOGRÁFICAS}

Alas, R. The impact of employee participation on job satisfaction during change process. Problems and Perspectives in Management, 5, (4), pp. 28-33; 2007.

Álvarez F. D. Satisfacción y fuentes de presión laboral en docentes universitarios de Lima Metropolitana; 2007.

Álvarez, G. y Miles, D. El papel de la empleabilidad en la satisfacción laboral de los trabajadores temporales. Revista Galega de Economía, 15, (002), pp. 1-20; 2006.

Andresen, M., Domsch, M. y Cascorbi, A. Working Unusual Hours and Its Relationship to Job Satisfaction: A Study of European Maritime Pilots. J Labor Res, 28, pp. 714734; 2007. 
Ayres, J. y Malouff, J. Problem-solving training to help workers increase positive affect, job satisfaction and life satisfaction. European Journal of Work and Organizational Psychology, 16, (3), pp. 279-294; 2007.

Crossman, A. y Harris, P. Job Satisfaction of Secondary School Teachers. Educational Management Administration Leadership, 34, pp. 29-46; 2006

D'Addio, A., Eriksson, T. y Frijters, P. An analysis of the determinants of job satisfaction when individuals' baseline satisfaction levels may differ, Applied Economics, 39, pp. 2413-2423; 2007.

Fisher, C., Harris, L., Kirk, S., Leopold, J. y Leverment, Y. The Dynamics of Modernization and Job Satisfaction in the British National Health Service. Review of Public Personnel Administration, 24, pp. 304-318; 2004.

Galup, S., Klein, G. y Jiang, J. The impacts of job characteristics on is employee satisfaction: a comparison between permanent and temporary employees. Journal of Computer Information Systems, pp. 58-68; 2008.

Gibson, J., Ivancevich, J. y Donelly, J. Fundamentos de dirección y administración de empresas. Colombia: McGraw Hill; 2001.

Hancer, M. y George, T. Job Satisfaction of Restaurant Employees: An Empirical Investigation Using the Minnesota Satisfaction Questionnaire. Journal of Hospitality \&amp Tourism Research, 27, pp. 85-100; 2003.

Lapierre, L. y Hackett, R. Trait conscientiousness, leader-member exchange, job satisfaction and organizational citizenship behavior: A test of an integrative model. Journal of Occupational and Organizational Psychology, 80, pp. 539-554; 2007.

Lee, Y. y Chang, H. Relations between Team Work and Innovation in Organizations and the Job Satisfaction of Employees: A Factor Analytic Study. International Journal of Management, 25, (3), pp. 732- 739; 2008.

Maslow, A. Motivation and Personality. New York: Harper \& Row Publishers; 1954.

Mason, C. y Griffin, M. Grouptask Satisfaction: Applying the Construct of Job Satisfaction to Groups. Small Group Research, 33, pp. 271-312.; 2002.

Morgan, G. Images of organization. (2 Ed.). USA: Sage; 1997. 
Morillo, I. Nivel de satisfacción del personal académico del Instituto Pedagógico de Miranda José Manuel Siso Martínez en relación con el estilo de liderazgo del jefe del departamento... Sapiens, 7, (001), pp. 43-57; 2006.

Moynihan, D. y Pandey, S. Finding Workable Levers Over Work Motivation: Comparing Job Satisfaction, Job Involvement and Organizational Commitment. Administration \&amp Society, 39, pp. 803-832; 2007.

Paik, Y., Praveen, K. y Shim, W. The relationship between perceived compensation, organizational commitment and job satisfaction: the case of Mexican workers in the Korean Maquiladoras. Int. of Human Resource Management, 18, (10), pp. 1768$1781 ; 2007$.

Rode, J. Job satisfaction and life satisfaction revisited: A longitudinal test of an integrated model. Human Relations, 57, pp. 1205-1230; 2004.

Rasouli A. Hossenian S. Dokanee F. Relación entre la resistencia y la satisfacción laboral y el estrés entre el personal y los miembros del cuerpo docente de la Universidad Islámica Azad de Mahabad Aras Rasouli. Iran; 2012.

Sendoya, S. Nivel de satisfacción de la médica egresada de la Universidad del Valle, Calí, Colombia. Colombia Médica, 31, (003), pp. 118-121; 2000.

Werther, W. y Davis, K. Administración de recursos humanos. El capital humano de las empresas. (6 Ed.). México: McGraw Hill; 2008.

Wright, B. y Davis, B. Job Satisfaction in the Public Sector: The Role of the Work Environment. The American Review of Public Administration, 33, pp. 70-90; 2003.

Wright, T. y Bonett, D. Job Satisfaction and Psychological Well-Being as Nonadditive Predictors of Workplace Turnover. Journal of Management, 33, pp. 141-160; 2007.

Alava, C.T. Psicología Clínico - Laboral. Lima: Editorial San Marcos, Segunda edición; 1984.

Flores García Rada, J. El Comportamiento Humano en las Organizaciones. Lima: Universidad del Pacífico; 1992.

García Alvarez, A.I. y Ovejero Bernal, A. Feedback Laboral y Satisfacción. España: Universidad de Oviedo; 2000. 
Kittleson, Ch. Identifying And Correcting Unsafe Employee Attitudes And Behavior. EEUU: Supervisor's Safety Update; 1996.

Robbins, S.P. Comportamiento Organizacional. México: Prentice Hall, Octava edición; 1998.

Van Haller Gilmer, B. Tratado de Psicología Empresarial, Tomo I. España: Ediciones Martínez Roca S.A; 1976.

Werther, W.B. y Davis, K. Administración de Personal y Recursos Humanos. México: McGraw Hill; 1996.

Wexley, K.N. y Yukl, G.A. Conducta Organizacional y Psicología del Personal. México: Compañía Editorial Continental S.A.; 1990. 